\title{
Una propuesta para estimar la población indígena en México a partir de los datos censales
}

\author{
Eric Janssen* \\ Regina Martínez Casas**
}

La estadística es aquella disciplina que permite decir que, cuando mi vecino tiene dos autos y yo ninguno, ambos tenemos un auto.

G. B. Shaw

En México la definición de la población indígena a partir de los datos de las encuestas suele restringirse a dos preguntas de carácter individual, la primera sobre el reconocimiento del uso de una lengua indígena, y la segunda sobre la autoadscripción étnica. Resulta cuestionable la validez de tal metodología debido a los riesgos de subestimación que induce. En este trabajo se propone una corrección de dicho cálculo al introducir el factor familiar, que permite mostrar de manera más clara la distribución de la población indígena en el país.

Palabras clave: indígenas, censo, población, vivienda.

\section{Introducción}

Uno de los puntos nodales en el uso de las estadísticas es la definición de las categorías a cuantificar. Bourdieu (1997) y Le Bras (1998a y 1998b), entre otros, han reconocido la importancia -y a menudo la controversia- de la delimitación de las categorías analizadas, producto de los prejuicios presentes en muchas de las representaciones sociales y de las preocupaciones políticas circunstanciales. Esto limita o sesga las respuestas, e impide que funcionen simplemente como indicadores sociales.

Una ilustración concreta de esta controversia en el contexto mexicano es el tratamiento de la información respecto al mundo indígena.

\footnotetext{
*Investigador del Centro Francés de Estudios Mexicanos y Centroamericanos, CEMCA, y del Centro de Investigaciones y Estudios Superiores en Antropología Social, CIESAS Occidente. Correo electrónico: janssen@ciesasoccidente.edu.mx.

** Investigadora del Centro de Investigaciones y Estudios Superiores en Antropología Social, CIESAS Occidente.
} 
Como numerosos países del continente americano, México encara el desafío de definir y luego cuantificar esas poblaciones, o por lo menos hacer estimaciones al respecto. Esta meta ha sido largamente obstaculizada por razones históricas, en particular luego de la Revolución de 1917 y de la posterior voluntad de fomentar una identidad nacional mestiza (para un análisis general de las reflexiones al respecto véase Bataillon et al., 1988 y Gamio, 1916 para el caso mexicano) que llevó a minimizar la diversidad cultural del país mediante la imposición de un modelo escolar único, entre otros criterios. Tanto que hasta el presente no existe un acuerdo significativo que permita establecer una metodología generalmente aceptada para definir dichas poblaciones y su volumen.

En este estudio se presenta una nueva metodología simple de cálculo para definir a la población indígena, apoyándose en las técnicas utilizadas con anterioridad. Se ilustran y examinan las ventajas y limitaciones de esta propuesta.

\section{La medición de la población indígena en México}

Sigue vigente el debate en México respecto a cómo definir lo que se ha denominado población indigena. Aunque la metodología no ha llegado a un consenso, la comunidad de investigadores está de acuerdo en asumir, dado el método de cálculo actual, una subestimación de esta población. Los problemas metodológicos derivan de una definición exclusivamente lingüística, pese a la ausencia de consenso sobre el número de idiomas indígenas que se hablan en el país (81 según De la Vega E., 2001; 91 según el INEGI, 2002; más de 100 según el Smithsonian Institute, etc.). Además aparece el fenómeno de la creciente migración y de que las segundas y terceras generaciones crezcan fuera de las comunidades de origen, lo que implica que cada día más niños no adquieran el idioma paterno, o que éste pase a ser su idioma secundario. Asimismo influyen las actitudes de desprecio por las lenguas indígenas producto del histórico desprestigio al que han estado expuestas. Consecuentemente, en el marco del XII Censo de Población y Vivienda (CPV de aquí en adelante), el INEGI planteó una segunda pregunta relativa al autorreconocimiento étnico. No negamos que esto representó una mejoría indudable respecto a la definición lingüística, pero quedan todavía al menos dos limitantes: la primera es que los niños menores de cinco años siguen fuera del conteo, y la segunda que 
la declaración de pertenencia obedece a motivos estrictamente subjetivos.

Asumimos en consecuencia que si para responder a esta pregunta se emprende una mejor estimación de la población indígena del país, en tal respuesta todavía permanece el sesgo que produce una subestimación de su tamaño. ${ }^{1}$

\section{Propuesta}

Nuestra propuesta sigue un doble eje que se basa en: 1) una ampliación de la definición, y 2) la traslación de una escala individual hacia otra en que se incluye el hogar. Sea $\mathrm{N}$ una población total compuesta de i individuos. Matemáticamente se escribe:

$$
\mathrm{N}=\sum_{\mathrm{i}=1}^{\mathrm{n}} \mathrm{i}, \mathrm{i}=1,2,3, \ldots, \mathrm{n}
$$

Asumimos que la población $\mathrm{N}$ se desagrega en solamente dos grupos: no indígenas e indígenas:

$$
\mathrm{N}=\mathrm{N}_{(\mathrm{ni})}+\mathrm{N}_{(\mathrm{i})}
$$

Es decir que se descartan los extranjeros y los residentes temporales, por una parte, y por otra la población de origen africano. ${ }^{2}$ Lo que importa ahora es definir la población indígena total $\mathrm{N}_{(\mathrm{i})}$. Hasta el censo de 1990 eran indígenas aquellas personas de cinco años o más que declaraban hablar un idioma oficialmente reconocido como indígena, es decir:

$$
\mathrm{N}_{(\mathrm{i})}=\sum_{\mathrm{i}=1}^{\mathrm{n}} \mathrm{h}_{(\mathrm{i})}
$$

${ }^{1}$ Se impuso el uso de la lengua como elemento exclusivo de la definición para no hablar de raza o cultura; se trata de un mecanismo para seguir defendiendo los criterios propios de una sociedad homogénea y mestiza.

${ }^{2}$ Como consecuencia del temor a desarrollar categorías de identificación étnica que pudieran parecer racistas, después de la Revolución de 1917 se eliminó la etiqueta negro o mulato en los conteos poblacionales para ubicarse en la gran categoría no indígena. A pesar de que la mezcla racial en México ha borrado muchos de los rasgos fenotípicos que en otras regiones del mundo se identifican como africanos, en algunas regiones de país como Guerrero y Veracruz todavía existen comunidades que no sólo se distinguen por su origen étnico, sino también porque reproducen algunos rituales y otros rasgos culturales provenientes de África y de las islas caribeñas (Aguirre Beltrán, 1984). 
A partir del censo de 2000 se introdujo la pregunta relativa a la autoadscripción para identificar a las personas de cinco años o más que se declaraban miembros de una etnia oficialmente reconocida, es decir:

$$
\mathrm{N}_{(\mathrm{i})}=\sum_{\mathrm{i}=1}^{\mathrm{n}} \mathrm{h}_{(\mathrm{i})}+\sum_{\mathrm{i}=1}^{\mathrm{n}} \mathrm{e}_{(\mathrm{i})}
$$

Nótese que estas definiciones excluyen a los niños de 0 a 4 años, y se centran exclusivamente en el individuo.

La idea es entonces aprovechar una posibilidad que ofrece la base de datos del último censo y adjuntar la dimensión familiar definida en este trabajo como el hogar. Así la población indígena se mediría como:

$$
\mathrm{N}_{(\mathrm{i})}=\sum_{\mathrm{i}=1}^{\mathrm{n}} \mathrm{h}_{(\mathrm{i})}+\sum_{\mathrm{i}=1}^{\mathrm{n}} \mathrm{e}_{(\mathrm{i})}+\Delta
$$

donde los dos primeros términos ubicados a la derecha de la fórmula siguen representando el eje individual y el $\Delta$ la dimensión familiar. La propuesta es extender a todos los integrantes del hogar la característica indígena si por lo menos uno de ellos se define como tal. No bastaría con preguntar sobre la práctica del idioma (o idiomas) que hablan las personas, o sobre su pertenencia étnica, sino que convendría estudiarlo junto con su hogar. Tal enfoque proporciona dos ventajas: la primera, ya mencionada, es que se aprovecha la capacidad de rebasar el enfoque exclusivamente individualista para reflexionar en términos de la conformación de los hogares como un paso necesario para conceptuar al grupo o a la comunidad $;^{3}$ la segunda es que se puede incluir a los niños menores de cinco años.

Tal propuesta se plantea a partir de algunos de los postulados de la teoría de la organización familiar y la reproducción ideológica asociada a ella, y reposa en una hipótesis de fuerte endogamia y reproducción interna de los indígenas. Hasta hoy no se han publicado estudios a gran escala relativos a las modalidades de unión de los indígenas que muestren este tipo de mecanismos de reproducción familiar. ${ }^{4}$ Sin embargo la literatura antropológica y etnológica tiende a validar tal

${ }^{3}$ Desde la teoría de la Ghestalt a fines del siglo XIX, se sabe que en las ciencias sociales un todo es más que la sola adición de las partes que lo componen. La propuesta permite rebasar esta limitación desde una perspectiva epistemológica.

${ }^{4}$ Para una revisión sobre los modelos familiares indígenas desde un punto desde una perspectiva etnográfica véase Robichaux, 2002. 
afirmación, en particular en las zonas rurales. Más aún, la creciente migración de los indígenas hacia las ciudades, y su integración en el sistema normativo característico del tejido urbano, no han conllevado a cambios en tales hábitos (véase Martínez Casas, 2002 y Bayona Escat, 2004). Víctimas del rechazo y del racismo cotidiano, los indígenas urbanos tienden a concentrarse en zonas marginales periféricas y a mantener sistemas de reproducción intracomunitaria muy marcados, que pasan entre otros por el mantenimiento de un contacto permanente con su comunidad de origen y por la endogamia matrimonial (Martínez Casas, 2001).

Sin embargo también otro factor se debe tomar en cuenta: los hogares que incluyen indígenas como empleados domésticos. ${ }^{5}$ En este caso no conviene extender la particularidad indígena a los demás miembros del hogar, sino que se debe considerar como una excepción cuyo cálculo se reduciría al aporte estrictamente individual ("habla o pertenece a una etnia”). Al final la población indígena estimada sería:

$$
\begin{aligned}
\mathrm{N}_{(\mathrm{i})} & =\sum_{\mathrm{i}=1}^{\mathrm{n}} \mathrm{h}_{(\mathrm{i})} \\
& +\sum_{\mathrm{i}=1}^{\mathrm{n}} \mathrm{e}_{(\mathrm{i})} \\
& +\sum_{\mathrm{i}=1}^{\mathrm{n}} \mathrm{H}_{(\mathrm{i})}-\sum_{\mathrm{i}=1}^{\mathrm{n}} \mathrm{d}_{(\mathrm{i})}=\Delta
\end{aligned}
$$

donde $\mathrm{H}_{(\mathrm{i})}$ representaría a los hogares considerados como indígenas excluyendo aquellos que declararon tener servidores domésticos indígenas $d_{(i)}$. Tal ecuación sugiere consecuentemente que las preguntas individuales respecto a la identidad étnica y más aún, a la práctica de un idioma indígena, deben extenderse a la unidad hogareña. Nótese que no se trata del primer intento por extender la delimitación de la población indígena al ámbito hogareño, puesto que el Conapo ${ }^{6}$ usó

${ }^{5}$ En las grandes ciudades del país como México, Guadalajara y Monterrey es común encontrar trabajadoras y trabajadores domésticos de origen indígena (Martínez Casas, 2000).

${ }^{6}$ El Consejo Nacional de Población (Conapo) se creó en 1973 con la finalidad de establecer políticas orientadas al control del crecimiento poblacional. 
un enfoque similar. ${ }^{7}$ Dados el racismo y la discriminación fuertemente extendidos en la sociedad mexicana e interiorizados por buena parte de esta población, proponemos como estrategia para el reconocimiento de la condición indígena formular una pregunta adicional: “¿Cuáles son los idiomas que se hablan en este hogar?”.

Otra posibilidad radica en una definición tal vez menos extensa. En vez de atribuir la característica indígena al conjunto de los miembros de un hogar si uno se declaró como tal (con la exclusión de los empleados domésticos), se propone extender dicha característica sólo cuando por lo menos una persona mayor de 15 años se defina como indígena, ${ }^{8}$ o el jefe del hogar o su cónyuge se definan como indígenas. Tal propuesta tiene la ventaja de recordar la importancia del lazo familiar, y no solamente el hogareño en la transmisión sociocultural de los atributos indígenas -postura implícitamente sostenida en la primera propuesta- al aislar los casos de los empleados domésticos que están en el seno de hogares considerados no indígenas.

Al final nos quedamos con dos definiciones basadas en la unidad doméstica y eventualmente en la relación de parentesco: una extensa y otra restringida. En la siguiente sección se ofrece una estimación de la población indígena en México a partir de los datos del CPV 2000.

\section{Aplicación}

Enseguida se propone un ejemplo comparativo que resulta de las diferentes definiciones incluidas en los datos del CPV nacional.

En el cuadro 1 se advierte que un simple cambio de definición permite casi duplicar la población considerada. Nótese también que la segunda definición extensa (el hogar con un miembro de 15 años y más) presenta cifras muy parecidas a la definición alternativa del Conapo enunciada en la nota 7. Semejante coincidencia nos lleva a evitar la distinción de estas dos definiciones de aquí en adelante. La

${ }^{7}$ Para alcanzar una población estimada de 12.7 millones de individuos a partir de los datos del CPV. Véase entre otros el discurso de presentación de la licenciada Elena Zúñiga Herrera en la inauguración del taller Metodologías para la Estimación de la Población Indígena, organizado por el Consejo Nacional de Población y el Instituto Nacional Indigenista y llevado a cabo el 7 de noviembre de 2002, que puede consultarse en: http://www.conapo.gob.mx/prensa/discursos2002_12.htm. Según esta misma metodología, la cifra alcanzó 13.2 millones para el año 2004.

${ }^{8}$ Proponemos los 15 años y no la mayoría de edad (18 años) por la precocidad con que los indígenas suelen unirse en pareja, tener hijos y eventualmente conformar un nuevo núcleo doméstico. 
CUADRO 1

Estimación de la población indígena según definición lingüística y étnica, México, 2000

\begin{tabular}{lrc}
\hline & & $\begin{array}{c}\text { Porcentaje } \\
\text { de la } \\
\text { población total }\end{array}$ \\
\hline Definición lingüística & Indígenas & 6.5 \\
Definición Censo 2000 & 7320520 & 7.7 \\
Definición restringida (jefe de hogar y/o cónyuge) & 11433196 & 11.8 \\
Definición extensa (15 años y más) & 12687307 & 13.1 \\
Definición del Conapo & 12700000 & 13.1 \\
\hline
\end{tabular}

FUENTE: CPV 2000.

definición adoptada para la realización del CPV 2000 muestra un cambio leve en la captación de la población indígena, pues incluye más de un millón de individuos respecto a los reconocidos conforme a la estricta definición lingüística; de esta manera se sostiene la validez metodológica de la pregunta sobre pertenencia étnica. Sin embargo al compararlo con nuestra propuesta todavía aparece una subestimación importante, lo que confirma la existencia tangible de una forma de enmascaramiento de la información por parte de los entrevistados y la necesidad de efectuar el conteo planteando nuevas preguntas. El cuadro 2 presenta las correlaciones entre las cuatro definiciones.

Los coeficientes resultan altamente significativos. La diferencia entre la definición restringida, que extiende la característica indígena al conjunto de los integrantes de los hogares en que el jefe o el cónyuge son indígenas, y la extendida, que atribuye la característica indígena al conjunto de los integrantes de los hogares que incluyen por lo menos a un indígena de 15 años y más, se centraría en nuestro argumento de la importancia de los lazos familiares para la reproducción lingüística y cultural y la transmisión de la identidad indígena a las nuevas generaciones. Respalda este argumento el que precisamente esta primera propuesta de definición alternativa alcance la mayor relación con la definición lingüística y étnica retenida por el CPV $(\rho=0.791, p<0.01)$.

En los porcentajes por grupos quinquenales de edad se advierte una diferencia entre los hombres y las mujeres indígenas. La mayor prevalencia de los tres primeros grupos en el caso masculino, sumada a una menor de los que se ubican en un rango entre 15 y 49 años, puede ser explicada de tres maneras posibles: 
1) La creciente migración laboral de tipo internacional, que suelen emprender los hombres jóvenes -tradicionales proveedores del bienestar material de sus hogares.

2) Una sobremortalidad de los sujetos mayores de edad de sexo masculino, agudizada a partir de los 50 años. A esta edad aparece una evidente desigualdad entre los hombres y las mujeres que se suele explicar porque ellos se exponen a los llamados "comportamientos riesgosos" (consumo de tabaco, alcohol, actividades laborales más exigentes o peligrosas, etcétera).

3) Una tendencia de la población masculina a no declarar su identidad indígena. Tal diferencia por género, si resulta válida, merecería una investigación más profunda..$^{9}$

En la gráfica 1 es posible comparar la distribución por edad de la población indígena según las dos definiciones, en contraste con la población no indígena. ${ }^{10}$

La población indígena aparece como sensiblemente más joven que la no indígena, con una mayor prevalencia en el grupo de edad de 0 a 14 años. La principal razón de tal diferencia es un retraso en la etapa final de la transición demográfica, con una reducción más tardía de la fecundidad (y de menor intensidad) que las demás poblaciones en

CUADRO 2

Correlaciones entre varias definiciones de la población indígena, México, 2000

\begin{tabular}{lcccc}
\hline & $\begin{array}{c}\text { Definición } \\
\text { del CPV }\end{array}$ & $\begin{array}{c}\text { Definición } \\
\text { restringida }\end{array}$ & $\begin{array}{c}\text { Definición } \\
\text { extensa }\end{array}$ & $\begin{array}{c}\text { Definición } \\
\text { del Conapo }\end{array}$ \\
\hline Definición del CPV & 1.000 & & & \\
Definición restringida & $0.791^{*}$ & 1.000 & & \\
Definición extensa & $0.774^{*}$ & $0.959^{*}$ & 1.000 & \multirow{2}{*}{0.000} \\
Definición del Conapo & $0.777^{*}$ & $0.958^{*}$ & $0.986^{*}$ & \multirow{2}{*}{1.000} \\
\hline
\end{tabular}

$* \mathrm{p}<0.01$

FUENTE: CPV 2000.

${ }^{9}$ En la investigación de campo que se llevó a cabo en las tres mayores concentraciones urbanas del país, las ciudades de México, Guadalajara y Monterrey, fue más frecuente que las mujeres adultas declararan su origen indígena a que lo hicieran los hombres o los niños. Esto se puede deber a que las madres son "responsables" de la transmisión de las normas y valores culturales y por ello sienten menor vergüenza ante la discriminación de la que suelen ser objeto los indígenas en México.

${ }^{10}$ Definida por la población promedio según las dos definiciones consideradas. 


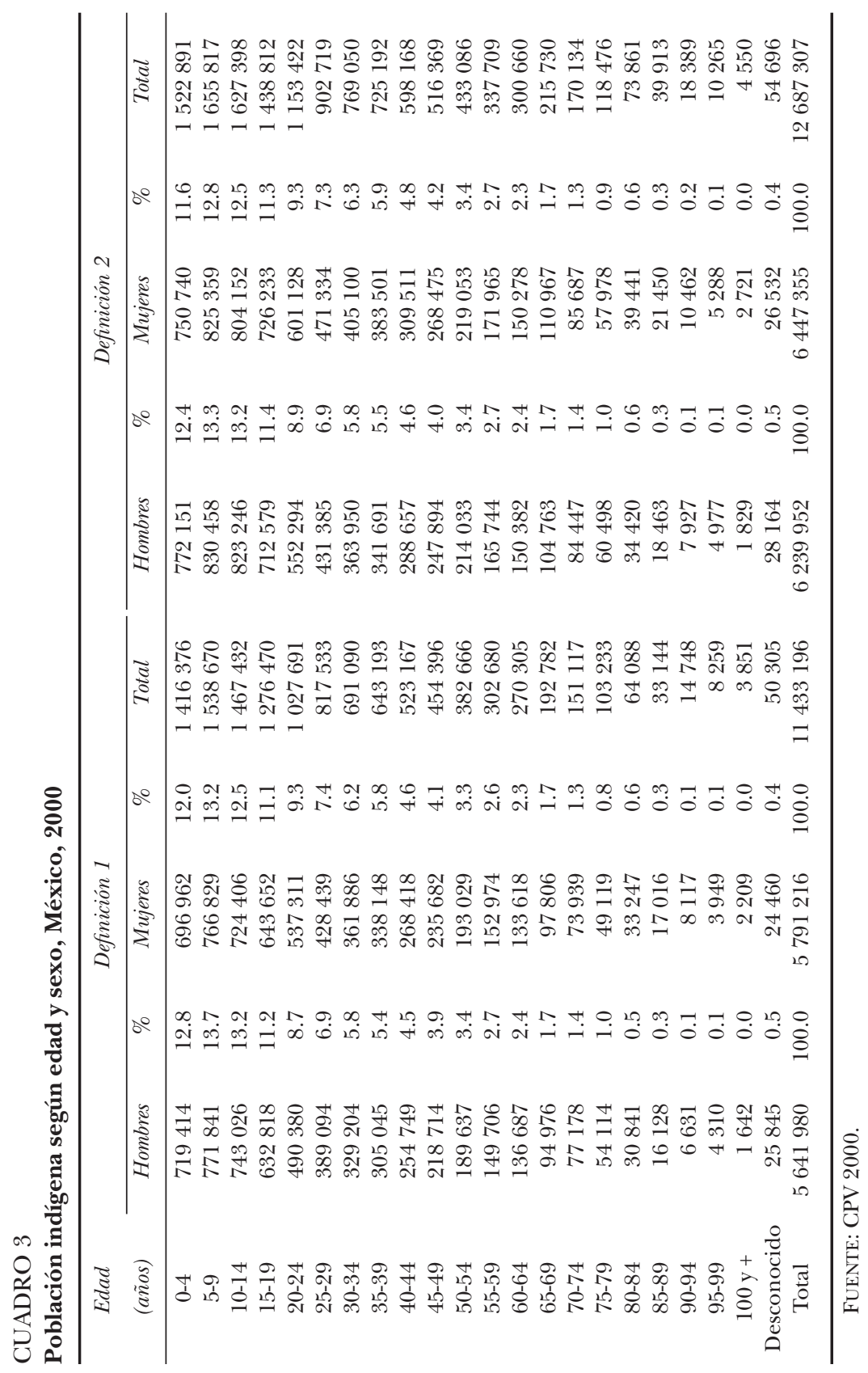


GRÁFICA 1

Distribución de la población indígena y no indígena por grupos de edad, México, 2000

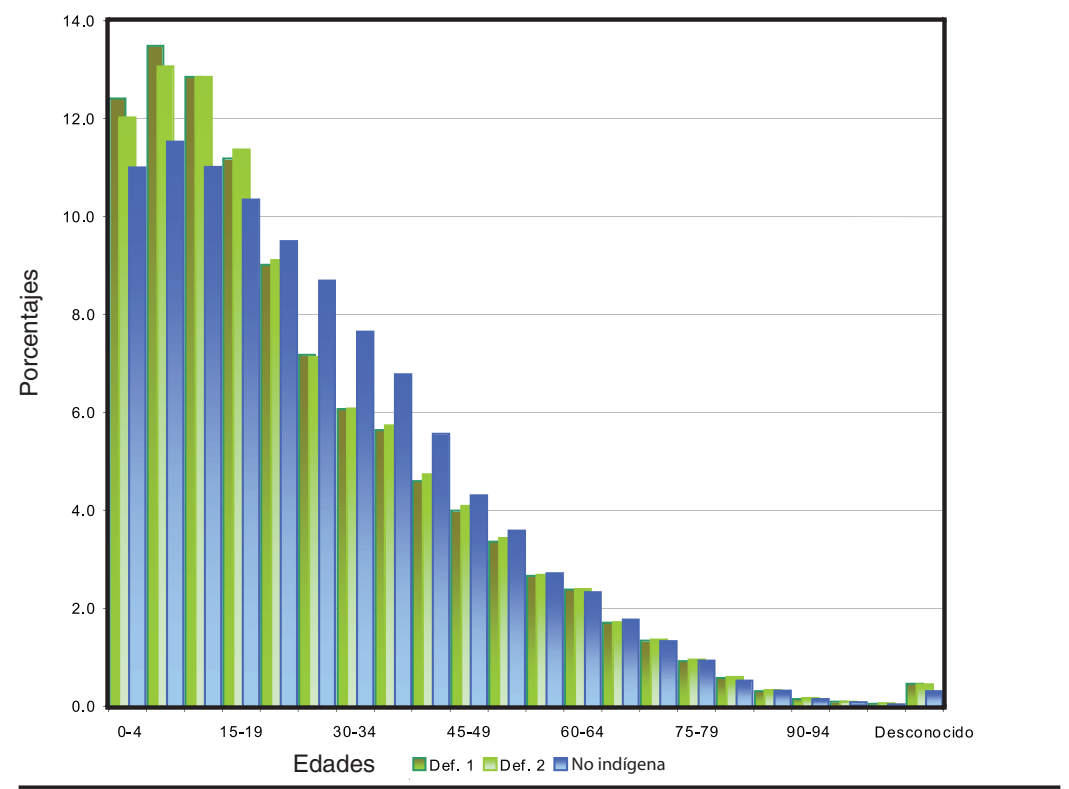

FUENTE: CPV 2000.

México. Cabe recordar que la reducción de la fecundidad en el país se originó en primer lugar en las ciudades, espacios sociales favorables a la emergencia de nuevas opiniones y oportunidades, y a la adopción de nuevos patrones de vida, donde hay una mayor oferta médica, para luego ir extendiéndose poco a poco hacia las zonas más aisladas. Confirman tal argumento los datos del cuadro 4.

A lo largo de la historia la población indígena se ha ubicado en zonas rurales, y hoy día la mitad está ahí. Si se le agregan los individuos que viven en zonas semiurbanas (de 2500 a 14999 habitantes), se alcanza $70 \%$ de la población indígena total, que habita en áreas con altos índices de marginación (Conapo, 2001). ${ }^{11}$ Sin embargo el CPV da cuenta de un fenómeno de migración hacia zonas urbanas que ha sido cada vez más intenso a partir de la segunda mitad del siglo XX.

${ }^{11}$ Correlación de 0.31 con la definición restringida $(\mathrm{p}<0.01)$ y de $0.27(\mathrm{p}<0.01)$ con la definición extendida. 
CUADRO 4

Población indígena según dos definiciones alternativas por tamaño de la localidad de residencia, México, 2000

\begin{tabular}{lrrrr}
\hline Tamaño de la localidad & Definición 1 & \multicolumn{1}{c}{$\%$} & Definición 2 & $\%$ \\
\hline Menos de 2500 habs. & 5886779 & 51.5 & 6165568 & 48.6 \\
2500 a 14999 habs. & 2298824 & 20.1 & 2462677 & 19.4 \\
15000 a 19999 habs. & 159209 & 1.4 & 183620 & 1.4 \\
20000 a 49999 habs. & 652829 & 5.7 & 729345 & 5.7 \\
50000 a 99999 habs. & 249061 & 2.2 & 294830 & 2.3 \\
100000 a 499999 habs. & 1122970 & 9.8 & 1410818 & 11.1 \\
500000 y más habs. & 1063524 & 9.3 & 1440449 & 11.4 \\
Total & 11433196 & 100.0 & 12687307 & 100.0 \\
\hline
\end{tabular}

FUENTE: CPV 2000.

Llama la atención que los indígenas presenten un patrón particular al integrarse directamente en los tejidos urbanos más densamente poblados.

En el cuadro 5 se presenta la distribución de la población indígena por estado.

Solamente en dos casos la población indígena rebasa 50\%: Yucatán y Oaxaca. Suele encontrarse mayormente en los estados del sureste de la República, en las zonas donde el desarrollo económico se ha centrado en el sector primario y es más reducida la inversión de capitales. Por el contrario, son escasos los indígenas que suelen ser subrepresentados en los estados asociados a la zona histórica de migración a Estados Unidos (parte occidental del país), y en los que alojan a las tres mayores conurbaciones.

\section{Discusión}

Hemos presentado aquí una metodología alternativa para definir y estimar la población indígena a partir de los datos del Censo Mexicano de Población y Vivienda del año 2000. Después de plantear el método de cálculo oficial centrado en las declaraciones de los individuos, y de subrayar los consecuentes problemas de subestimación de dicha población, se propuso aprovechar una valiosa aportación del censo y centrarse en la conformación de los hogares. Como complemento se 
CUADRO 5

Población indígena en cada entidad federal según dos definiciones alternativas, México, 2000 (porcentajes)

\begin{tabular}{|c|c|c|}
\hline Entidad & Definición 1 & Definición 2 \\
\hline Aguascalientes & 0.7 & 0.9 \\
\hline Baja California & 4.6 & 5.8 \\
\hline Baja California Sur & 3.4 & 3.7 \\
\hline Campeche & 29.1 & 30.8 \\
\hline Coahuila & 0.6 & 0.8 \\
\hline Colima & 1.5 & 1.9 \\
\hline Chiapas & 32.2 & 33.3 \\
\hline Chihuahua & 6.1 & 7.3 \\
\hline Distrito Federal & 4.9 & 7.0 \\
\hline Durango & 1.7 & 2.7 \\
\hline Guanajuato & 1.1 & 2.0 \\
\hline Guerrero & 18.3 & 19.5 \\
\hline Hidalgo & 25.5 & 26.9 \\
\hline Jalisco & 1.7 & 2.6 \\
\hline México & 8.2 & 9.8 \\
\hline Michoacán & 6.5 & 7.4 \\
\hline Morelos & 5.4 & 6.6 \\
\hline Nayarit & 10.6 & 11.6 \\
\hline Nuevo León & 1.1 & 1.8 \\
\hline Oaxaca & 54.6 & 56.4 \\
\hline Puebla & 19.6 & 21.3 \\
\hline Querétaro & 3.6 & 4.6 \\
\hline Quintana Roo & 43.6 & 45.9 \\
\hline San Luis Potosí & 15.1 & 16.2 \\
\hline Sinaloa & 4.1 & 4.9 \\
\hline Sonora & 8.6 & 9.8 \\
\hline Tabasco & 9.1 & 10.4 \\
\hline Tamaulipas & 2.2 & 2.6 \\
\hline Tlaxcala & 7.6 & 9.2 \\
\hline Veracruz & 15.5 & 16.8 \\
\hline Yucatán & 64.2 & 67.1 \\
\hline Zacatecas & 0.4 & 0.7 \\
\hline Total & 11.8 & 13.1 \\
\hline
\end{tabular}

FUENTE: CPV, 2000. 
restringe el impacto de los hogares en donde los indígenas trabajan como empleados domésticos.

El cálculo se apoya en una hipótesis de endogamia que puede y debe vincularse con el fenómeno migratorio y otros procesos asociados a las nuevas conformaciones poblacionales de los grupos indígenas del país. Así, es factible imaginar que la primera generación que se hallara fuera de la comunidad tendería a mostrar una endogamia de casi $100 \%$, es decir una reproducción exclusiva en el marco de la etnia; los costos de adaptación (choque cultural, fricciones, auto y heterodiscriminación), y los conflictos (racismo, ostracismo, etc.) pueden fortalecer esta tendencia de reproducción interna y de repliegue sobre sí. Recordemos por ejemplo la referencia a la fuerte presión social para la unión matrimonial precoz.

Pero se puede suponer que dicha endogamia se debilitará en el transcurso del tiempo (segundas y terceras generaciones) con la adopción progresiva de nuevos sistemas normativos y de valores. Por otro lado, no se puede negar la presión de la lógica global de producción y consumo a la cual se deben adaptar los indígenas para sobrevivir. Este conjunto de factores da lugar a cambios y resignificaciones de modelos culturales y a la consecuente búsqueda de otras formas de vida en espacios más grandes que los comunitarios, como son las concentraciones urbanas. La reproducción tendería a pasar por una apertura hacia otras capas de la población, no necesariamente indígena. Sin embargo en los primeros encuentros con la población indígena migrante se advierte una permanencia -aunque resignificada-de sus mecanismos de reproducción socioeconómica, a corto y mediano plazos (véase Martínez Casas, 2002).

Esta novedosa propuesta teórico-metodológica permite pasar de la estricta definición individual, que puede estar dejando fuera de los conteos a los indígenas más jóvenes, entre otros que niegan su condición de hablantes, porque facilita su inclusión en los cálculos de este segmento poblacional. Con la finalidad de rebasar este problema se propuso preguntar en las encuestas “¿cuáles son los idiomas que se hablan en este hogar?". Tal pregunta introduce la cuestión hogareña previamente citada e incluye el contexto familiar y cultural que hasta el momento ha escapado a la definición individual. Además, como no se enfoca sobre un solo individuo, se espera minimizar el fenómeno de autocensura muy comúnmente observado en las respuestas sobre el origen étnico.

Obviamente, nuestras propuestas solamente podrán mostrar su 
efectividad tras practicar estudios de campo más generalizados, recordando la necesidad de un enfoque multidisciplinario (cuantitativo y cualitativo) para el análisis lo más holístico y sistémico posible de esta problemática compleja.

Más allá del simple conteo de población, la subrepresentación implica problemas de mayor alcance, como el bienestar de los grupos indígenas. El diseño y adecuación de políticas sociales dirigidas a poblaciones de alta vulnerabilidad incluye la migración de los indígenas a las zonas urbanas, pero dada esta subrepresentación son muy pocos los programas expresamente diseñados para atender su inserción en las ciudades. Por otro lado, la particular organización social de los indígenas de la que hablamos ya escuetamente y que tiene que ver con los patrones matrimoniales y parentales endógamos, la creación de redes solidarias, y la reproducción resignificada de los modelos culturales indígenas en la ciudad, obligan a formular consideraciones especiales al ofrecer programas sociales a esta población. El hecho de ignorar su dimensión no contribuye a garantizar su éxito. La subrepresentación implica también una minimización de la importancia de la diversidad lingüística y cultural del país, lo cual contribuye a reforzar la histórica exclusión de la población indígena de la sociedad nacional.

\section{Bibliografía}

Aguirre Beltrán, G. (1984), La población negra en México, México, Fondo de Cultura Económica.

Bataillon, C. et al. (1988), Indianidad, etnocidio e indigenismo en América Latina, México, Instituto Indigenista Interamericano/CEMCA.

Bayona Escat, E. (2004), "Niños indígenas trabajadores: los casos de los purépechas y los mixtecos en Guadalajara", seminario de Presentación de los Primeros Resultados del Proyecto Niños Indígenas en Escuelas Urbanas: el Caso de Jalisco, Guadalajara, CIESAS Occidente, 28 de octubre.

Bourdieu, P. (1997), Les usages sociaux de la science, París, INRA.

De la Vega Estrada, S. (2001), Índice de desarrollo social de los pueblos indígenas, México, INI/PNUD.

Conapo (2001), Índices de marginación 2000, $1^{\text {a }}$ edición, diciembre, México.

Gamio, M. (1916), Forjando patria: pronacionalismo, México, SEP. INEGI (2002), XII Censo de población y vivienda, Aguascalientes.

Le Bras, H. (1998a), "La confusion des origines", trabajo presentado en el coloquio del INSEE Satistique Sans Conscience N'est que Ruine, París, 4 de noviembre. 
(1998b), "Les Français de souche existent-ils?”, trabajo presentado en el coloquio del INSEE Statistique Sans Conscience N'est que Ruine, París, 4 de noviembre.

Martínez Casas, R. (2002), "La comunidad moral como comunidad de significados: el caso de la migración otomí en la ciudad de Guadalajara”, Alteridades, vol. 23, pp. 125-139.

- (2001), Una cara indígena de Guadalajara: la resignificación de la cultura otomi en la ciudad, tesis de doctorado en Ciencias Antropológicas, México, Universidad Autónoma Metropolitana, Unidad Iztapalapa.

(2000), "La presencia indígena en Guadalajara: los vendedores de la Plaza Tapatía”, en R. Rojas y A. Hernández (coords.), Rostros y palabras. El indigenismo en Jalisco, México, Instituto Nacional Indigenista, pp. 41-62.

Robichaux, D. (2002), "El sistema familiar mesoamericano: testigo de una civilización negada”, en G. de la Peña y L. Vázquez León (coords.), La antropología sociocultural en el México del milenio. Búsquedas, encuentros y transiciones, México, CFE/INI/CONACUlTA, pp. 107-161 (Biblioteca Mexicana).

Serrano Carreto, E., A. Embriz Osorio y P. Fernandez Ham (coords.) (2002), Indicadores socioeconómicos de los pueblos indígenas de México, 2002, México, INI/PNUD/Conapo. 